\title{
Long-Term Repellency of Liquids by Superoleophobic Surfaces
}

\author{
Periklis Papadopoulos, Doris Vollmer, and Hans-Jürgen Butt \\ Max Planck Institute for Polymer Research, Ackermannweg 10, D-55128 Mainz, Germany*
}

(Dated: October 27, 2016)

\begin{abstract}
Applications of superoleophobic surfaces depend on the stability of the air cushion formed under liquid drops. To analyze the longevity of air cushions we used reflection-interference contrast microscopy (RICM) for drops on a porous fractal-like structure of sintered nanoparticles. RICM permits us to monitor the height of the air cushion with nanometer resolution. Whereas the air cushion under all investigated liquids was stable on a time scale of a few seconds to minutes and liquids rolled off, liquids with low surface tension penetrated the coating on the time scale of hours and longer. The penetration speed showed a power law dependence on time, $d z / d t \sim t^{p}$, the exponent $p$ varying from -0.5 to -1.2 . Thus, penetration is qualitatively different from the Lucas-Washburn law that governs spontaneous capillary filling of porous structures.
\end{abstract}

Surfaces with low wettability for both water and organic liquids are important for everyday applications and industry, including self-cleaning [1], anti-icing [2], and anti-fouling [3-5]. Micro- or nanostructured surfaces can show superior liquid repellency to smooth surfaces. Imbibition of liquids into rough or porous surfaces can be prevented by structuring the surfaces with protrusions in such a way that the liquid wets only the topmost part of protrusions and air is entrapped underneath $[6,7]$. On structured surfaces the apparent contact angle of drops is defined by the shape of the drop on a length scale much larger than the protrusions, usually several $\mu \mathrm{m}$. A commonly used term for surfaces that allow for apparent contact angles greater than $150^{\circ}$ and easy roll off of organic liquids is "superoleophobic," as organic liquids are generally referred to as "oils" [8]. In air these surfaces are also superhydrophobic [9].

Several superoleophobic surfaces are highly porous. In contrast to wettable porous materials, little insight exists on the penetration of liquids into nonwettable porous surfaces. Porous surfaces either repel a liquid, such as hydrophobic soils $[10,11]$, or the liquid imbibes the surface. The wetting state with trapped air was first described by Cassie and Baxter for water [12]. A liquid can also wet a rough solid completely, forming the Wenzel state [13]. In addition to geometry, also the wettability of the material, and external pressure $[14,15]$ determine which of the two is the thermodynamically stable state. Practical applications also require a Cassie state that is stable against impact [16-18], vibrations [19], chemical degradation, mechanical damage [20-22], and contamination for as long as possible. Often the drop does not reach the thermodynamically stable state and stays in a metastable state. Little is known on the long-term stability of the Cassie state for drops on porous superoleophobic surfaces. A detailed understanding of the dependence of the penetration depth on time is helpful to control imbibition of liquids into porous surfaces.

Here, we study the long-term stability of the Cassie state of a superoleophobic surface for nonpolar liquids. Wetting states where some nanoparticles are completely wetted and others not or only partially are termed as mixed Cassie-Wenzel states [Fig. 1(h)]. As a reference surface we choose a soot-templated structure comprising a fractal-like network of self-assembled nearly spherical nanoparticles $[23,24]$ [Figs. 1(a) and 1(b)]. Both the solid and the gas phase are continuous, the gas phase comprising about $90 \%$ of the volume. Nonpolar liquids form contact angles $\theta<90^{\circ}$ on flat surfaces of this material, so the material is "hygrophilic" [9], but the rough structure allows nearly any liquid to form spherical drops. By means of RICM, we monitor the position of alkane-air interfaces with a height resolution better than $20 \mathrm{~nm}$. Measuring the whole range of liquid $n$ alkanes allows us to finely vary the energy barrier for a Cassie-to-Wenzel transition, while maintaining constant surface tension during the experiment, unlike the commonly applied ethanol-water mixtures $[25,26]$. A theoretical model of sintered spheres [Figs. 1(e) and 1(f)] shows that thermal fluctuations or vibrations can possibly trigger penetration of liquid into the nanostructures.

Drops of water and $n$-hexadecane were gently deposited with a syringe onto the superoleophobic surface [Fig. 3(a)]. Confocal microscopy [27] verified that both drops were in the Cassie state, staying at the top of the porous superoleophobic surface [Figs. 1(c) and 1(d)]. Water, poly(dimethyl siloxane) (PDMS, Sigma-Aldrich, viscosity $100 \mathrm{cSt}$ ) and all liquid $n$-alkanes from $n$-octane and longer did not reach the completely wetted Wenzel state for at least several hours. In Fig. 2 both advancing and receding apparent contact angles on the superoleophobic surface on a length scale of micrometers $\Theta_{a d v}^{a p p}, \Theta_{\text {rec }}^{a p p}$ and their respective values $\theta_{a d v}, \theta_{\text {rec }}$ on a flat glass with the same surface chemical composition are shown. The contact angles $\theta_{a d v}, \theta_{\text {rec }}$ are termed the material's advancing and receding contact angles. All apparent advancing contact angles were indistinguishable from $180^{\circ}[29]$. The differences in superoleophobicity are reflected by the receding contact angles that were as low as $120^{\circ}$ for $n$-octane, implying that the wetted area fraction is larger and that the distance between wetted particles decreased $[26,30]$. We determined $\Theta_{a d v}^{a p p}, \Theta_{r e c}^{a p p}$ by confo- 


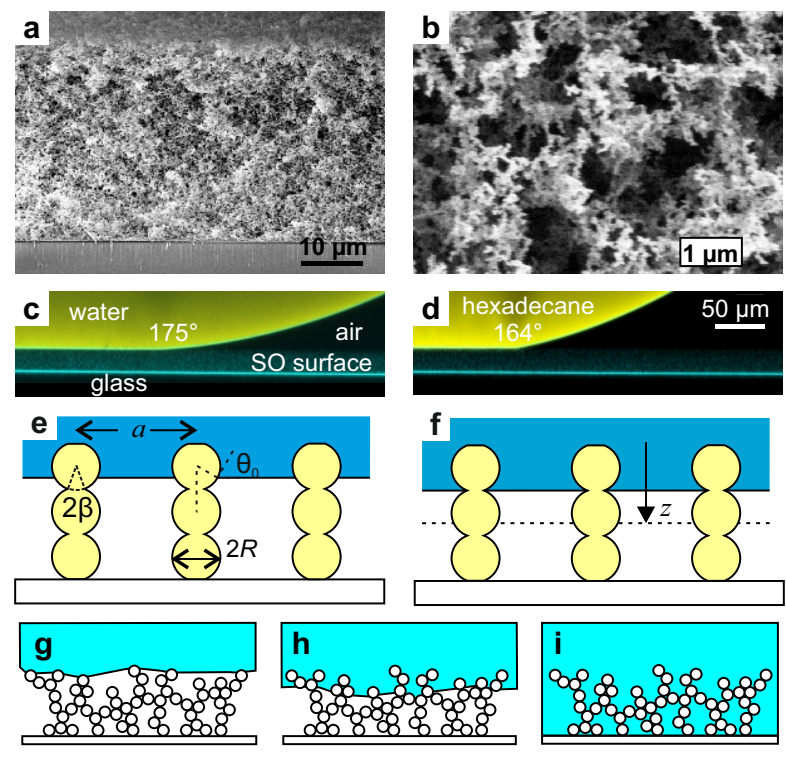

FIG. 1. Superoleophobic surfaces. (a) A coating based on soot templating. Scanning electron microscope (SEM) side view image of a cross section. Candle soot deposited on glass was covered with a silica shell about $20 \mathrm{~nm}$ thick, soot was combusted at $500^{\circ} \mathrm{C}$ and the remaining transparent silica shell was hydrophobized. (b) Top view SEM image. (c) Confocal laser scanning microscope (CLSM) vertical cross section of a fluorescently labeled water drop (yellow) on this coating. Reflection shown in cyan. (d) A fluorescently labeled $n$ hexadecane drop on the same coating. (e) Model of sintered spheres for calculation of the energy barrier for liquid penetration. Equilibrium position, where the liquid-air interface forms the Young's contact angle $\theta_{0}$ with the solid. (f) Local energy maximum and next equilibrium position (dashed line). Depth $z$ is measured from the top of the pillars. (g) Cassie wetting state of a water drop on this coating. (h) A mixed Cassie-Wenzel state and (i) the Wenzel state.

cal microscopy, by increasing and decreasing the volume of sessile drops with a syringe and measuring simultaneously vertical $(x z)$ slices, similar to Figs. 1(c) and 1(d), taking care that the apparent contact line moved [31]. Profiles were fitted with a circular arc (Fig. S2). In the case of apparent angles lower than about $130^{\circ}$ we used a conventional camera (Dataphysics Instruments $\mathrm{GmbH}$, OCA 20) [32].

The same confocal microscope with a dry objective of low numerical aperture $(10 \times / 0.40$ dry, Nikon) was employed for RICM. Interference is generated from reflections at the lower side of the water drop and the top side of the glass substrate. RICM images of water drops resembled interference fringes from two nearly parallel surfaces [Figs. 3(a) and 3(b)]. Over the whole apparent contact diameter of $250 \mu \mathrm{m}$ the spacing between the interfaces varied by only one wavelength $(\lambda=473 \mathrm{~nm})$, most likely due to a different thickness of the superoleophobic layer.

Alkane drops carefully deposited onto the surface

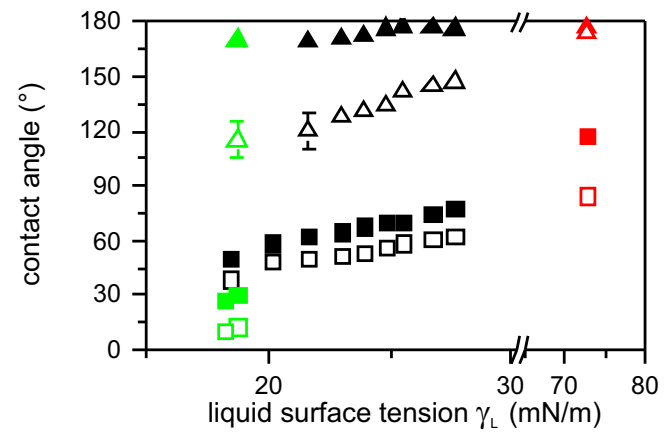

FIG. 2. Material's contact angles $\theta_{a d v}, \theta_{r e c}$ (squares) and respective apparent contact angles $\Theta_{a d v}^{a p p}, \Theta_{r e c}^{a p p}$ on the superoleophobic surface (triangles). Advancing and receding values shown by full and hollow symbols, respectively. Water, alkanes, PDMS are marked red, black, green, respectively. Unless explicitly shown, the error bars are smaller than the symbols.

showed a similar interference pattern just after deposition [n-decane in Fig. 3(c)]. In contrast to water, this pattern changed with time. Fringes shifted, showing that the thickness of the air layer decreased. In addition, the pattern became more irregular and less planar. $n$ decane slowly wetted the fractal-like structure of the superoleophobic coating [Figs. 3(e) and 3(g)]. As it creeped into the layer, the drop passed through a series of mixed Cassie-Wenzel wetting states [Fig. 1(h)]. We found no indication for condensation of drops that were larger than the resolution of the confocal laser scanning miroscope, $\sim 200 \mathrm{~nm}$, in the porous structure. On the contrary, for water the lower liquid-air interface remained constant despite having higher vapor pressure than all alkanes from $n$-octane and higher (Table S1). Furthermore, PDMS penetrated as fast as dodecane, even though it does not evaporate.

To analyze the process of impalement we measured the increase in penetration depth $z$ for each pixel versus time [Fig. 1(f)]. Hereby, the initial position at $t=0$ is defined as zero; it is different for every pixel. With time, the reflected intensity from each pixel changed [Fig. 3(d)] several times from high to low and back, each cycle corresponding to half a wavelength; the reflected light from the drop-air interface undergoes a phase shift of $180^{\circ}$. Depth as a function of time for this pixel is shown in Fig. 3(d). By repeating this procedure for every pixel maps of the penetration depth were obtained [Figs. 3(f) and 3(h)]. Depth continued to increase even after several hours.

Wetting proceeded in a spatially and temporally inhomogeneous way, as the variation of the depth at different positions of the same drop shows [Fig. 4(a)]. For $n$-decane, the penetration depth varied by up to 0.2 $\mu \mathrm{m}$ over areas of $5 \times 5 \mu \mathrm{m}^{2}$ after one hour. This shows that there is cooperativity between neighboring protrusions [33]. The qualitative picture is similar at different 

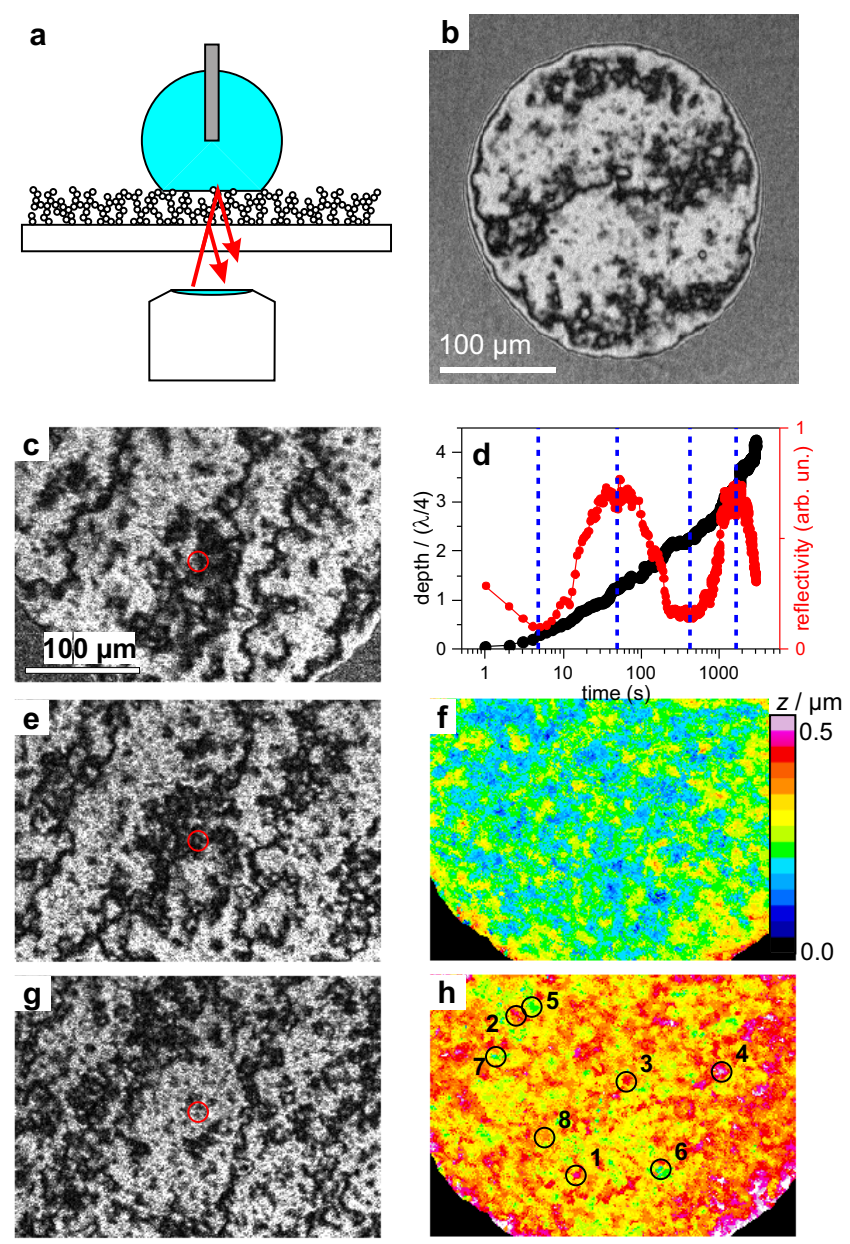

FIG. 3. Penetration depth of liquids on the superoleophobic surface extracted from RICM. (a) Interference from reflections at the glass-coating and coating-liquid interfaces. The volume of the drop was kept constant at $5 \mu \mathrm{l}$. (b) RICM image of a water drop on the same superoleophobic surface as Figs. 1 and 2. (c) RICM image of an $n$-decane drop 0.13, (e) 800, and (g) $2500 \mathrm{~s}$ after deposition. (d) Time dependence of reflected intensity of a pixel in the series of RICM images (red, marked with a circle in (c),(e),(g)). (f),(h) Penetration depth corresponding to (e),(g). Depth at $t=0$ is defined as zero. The points marked as 1-8 are used for analysis in Fig. $4(\mathrm{a})$.

spots. Therefore, we evaluated the mean depth, averaged over large areas $\left(>100 \times 100 \mu \mathrm{m}^{2}\right)$. The penetration velocity decreased with time and increasing alkane length [Fig. 4(b)]. Whereas $n$-heptane reached the Wenzel state within seconds, $n$-hexadecane needed about $24 \mathrm{~h}$ to penetrate $100 \mathrm{~nm}$. A height of $100 \mathrm{~nm}$ corresponds roughly to one diameter of the nanoparticles of the soot template. Stronger variations at short times are most likely due to the fact that the porous surface is less homogeneous at the top than somewhere in the middle.

For a quantitative evaluation, we calculated the penetration velocity as a function of time. The velocity does
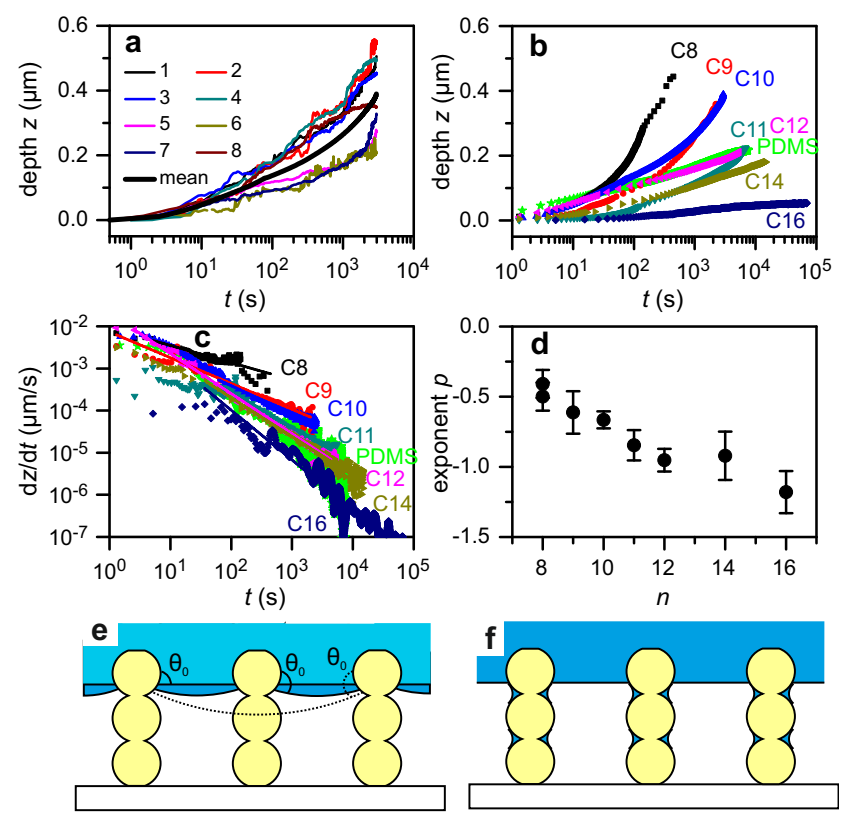

FIG. 4. Wetting of the same superoleophobic surface (Fig. 1 ) by different $n$-alkanes and PDMS. (a) Penetration depth $z(t)$ of $n$-decane at different spots of the surface for the same drop [Fig. 3(h)]. The mean of all eight curves is shown with the thick solid black line. (b) Time dependence of the average depth (averaged over $0.01 \mathrm{~mm}^{2}$ ) for different alkanes and PDMS. (c) Penetration velocity showing a power-law dependence on time after deposition. (d) Exponent $p$ of the power law dependence on the number of carbon atoms of the alkane. Error bars from three independent measurements. (e) Effect of Laplace pressure. A curved interface forms the angle $\theta_{0}$ with the solid at a lower position than a horizontal interface. At larger spacing, the effect is more pronounced (dotted line). (f) Capillary condensation at the neck.

not depend on our choice of zero depth [Fig. 4(c)]. It showed a power law dependence on time, $d z / d t \sim t^{p}, p<$ 0 . The exponent $p$ decreases with increasing alkane length [Fig. 4(d)]. For octane it is about -0.5 , similar to the power law dependence for wetting of cylindrical pores according to Lucas-Washburn law [34, 35]. For higher alkanes the exponent is lower, but still greater than -1 , except for hexadecane. For PDMS, we measured $-1.0 \pm 0.1$. This power law dependence shows that penetration is not expected to stop even after several days. It was impossible to extract the exponent by interference microscopy for $n$-hexane and $n$-heptane, as penetration was too fast.

The observed penetration process is different from classical imbibition in porous media. In spontaneous imbibition, as for example described by Lucas-Washburn $[34,35]$, the liquid flow is hindered by viscosity. In this case, velocity follows a power-law dependence on time with an exponent close to -0.5 , even though varying scaling laws may be found for the width of the rough wetting front [36-41]. In our case the velocities were so low that 
viscous dissipation was not the limiting factor. Moreover, velocities varied by several orders of magnitude, whereas the viscosity varied only by a factor of 10 from hexane to hexadecane $(0.31$ to $3.5 \mathrm{mPa}$ s, Table $\mathrm{S} 1[42,43])$. In addition, the Laplace pressure of the drop $P_{L}=2 \gamma_{L} / R$ is low, about $50 \mathrm{~Pa}$, as the drop radius is about $1 \mathrm{~mm}$. The characteristic distances between particles in the structure are on the order of $1 \mu \mathrm{m}$ or less, so the influence of the Laplace pressure is negligible. We conclude that the system passed through a series of metastable wetting states. If creeping imbibition is caused by individual small steps and thermal fluctuations, the creep velocity may be described similarly to the Molecular Kinetic Theory [44-47] by $d t / d t=\delta \nu \exp -\Delta G / k_{B} T$. Here, $\delta$ is the step width, $\nu$ is the attempt frequency, and $\Delta G$ is the energy barrier. As the measured penetration velocity depended on small changes of the material's contact angle $\theta_{a d v}$ (Fig. 2, Table S1), we expect that the energy barrier created by overhangs is probably only a few $k_{B} T$ and depends on the wettability of the material by the liquid.

We estimate this energy barrier by using the model of Figs. 1(e) and 1(f). Sintered silica particles are modeled as spheres with truncated tops and bottoms that form columns. The columns form a square array with a centerto-center distance of $a$. This model is similar to models of packed spheres previously used for the study of the wetting of soils. We make the approximation that the liquid-air interface is horizontal [25, 26]. At equilibrium the liquid forms the Young's contact angle $\theta_{0}$ with the solid [48]:

$$
\gamma_{L V} \cos \theta_{0}=\gamma_{S V}-\gamma_{S L}
$$

where $\gamma_{L V}, \gamma_{S V}, \gamma_{S L}$ are the liquid-vapor, solid-liquid and solid-vapor interfacial tensions, respectively. This is the state of minimum energy. The local energy maximum is at the sintering point [Fig. 1(f)], where the liquid-vapor area per column is maximal. Below and above this point the contact line would spontaneously advance or recede, respectively. Without considering fluctuations, imbibition would be spontaneous only when $\theta_{0}<\beta$, where $\beta$ is the angle defining sintering [Fig. 1(e)]. As interfacial energies are interrelated through Young's equation [Eq. (1)], the energy barrier per column is estimated by the differences of liquid-vapor and solid-liquid areas per column, $A_{L V}$ and $A_{S V}$ respectively, between the two states:

$$
\begin{aligned}
\Delta G= & \gamma_{L V} \Delta A_{L V}+\left(\gamma_{S L}-\gamma_{S V}\right) \Delta A_{S L} \\
= & \gamma_{L V}\left(\pi R^{2} \sin ^{2} \theta_{0}-\pi R^{2} \sin ^{2} \beta\right) \\
& -\gamma_{L V} \cos \theta_{0} 2 \pi R \cdot R\left(\cos \beta-\cos \theta_{0}\right) \\
= & \gamma_{L V} \pi R^{2}\left(\cos \beta-\cos \theta_{0}\right)^{2}
\end{aligned}
$$

The Young's contact angle is not directly measurable due to contact angle hysteresis, but it is commonly estimated by the mean cosine of $\theta_{a d v}, \theta_{\text {rec }}[49]$ :

$$
\cos \theta_{0}=\left(\cos \theta_{a d v}+\cos \theta_{r e c}\right) / 2
$$

Inserting structure dimensions consistent with SEM images $\left(\beta=50^{\circ}, R=50 \mathrm{~nm}\right)$ and typical wetting parameters of alkanes $\left(\theta_{0}=60^{\circ}, \gamma_{L V}=24 \mathrm{mN} / \mathrm{m}\right.$, Table S1) we get $\Delta G=3.4 \times 10^{-18} \mathrm{~J} \approx 10^{3} k_{B} T$ at $\mathrm{T}=300$ $\mathrm{K}$. The quadratic dependence of the energy barrier on $\cos \beta-\cos \theta_{0}$ can explain the speeding up of penetration with decreasing alkane length, as $\theta_{0}-\beta \rightarrow 0$ for decreasing alkane length (Table S1). The energy barriers can be reduced by several factors.

(i) As the superoleophobic surfaces do not consist of a regular structure, wetting proceeds by wetting preferably overhangs with a low energy barrier, that is a large value of $\beta$. Values of $\beta$ up to $70^{\circ}$ occur, according to SEM images, even though the average value may be lower [Figs. $1(\mathrm{c})$ and $1(\mathrm{~d})]$.

(ii) Small particles in cavities between larger structures enhance imbibition when the wetting front touches one of these particles [11, 25].

(iii) Two neighboring spherical structures are usually not perfectly aligned vertically. In general, they are aligned at a certain angle with the vertical line.

(iv) For volatile liquids, capillary condensation at the narrow point of a neck may occur, effectively increasing $\beta$ [Fig. 4(f)]. As alkanes are volatile compared to PDMS, this explains why they penetrate faster, despite having a higher material's contact angle. The real critical material's contact angle for this structure is about $30^{\circ}$ for PDMS, lower than the $50.73^{\circ}$ predicted for structures of close packed spheres [11]. The low solid fraction allows depinning to occur at single overhangs without affecting their relatively distant neighbors, in contrast to dense reentrant structures.

Besides thermal fluctuations, vibrations of the drop caused by depinning of the contact line or acoustic vibrations may also help to overcome the energy barrier. Finally, the Laplace pressure may also decrease the energy barrier. A curved liquid-air interface forms $\theta_{a d v}$ at a lower position as compared to a planar surface [Fig. 4(e)]. The position of the interface is lower for larger spacing between pillars, so the effect of the Laplace pressure increases. As the superoleophobic surface has a random structure, initially [Fig. 1(g)] the average spacing between wetted particles is much larger than the particle diameter. When the liquid creeps into the structure, the average spacing decreases [Fig. 1(h)]. The decreasing spacing and the increasing number of contact points also leads to higher damping of acoustic vibrations.

The Cassie state of liquids of high and low surface tension on soot-templated superoleophobic surfaces was stable on short time scales. However, a low roll-off angle and high apparent contact angles do not guarantee long-term stability. Even though for long chain alkanes the Cassie 
state is stable at least for days, volatile and nonvolatile liquids with low surface tension slowly penetrated the porous structure. The slow transition to the Wenzel state through mixed metastable wetting states resembles dynamic wetting and is possibly triggered by thermal fluctuations or drop vibrations. The results are also relevant for porous structures that are poorly wettable by a liquid. Instead of a sharp transition between nonwettability and imbibition at a critical material's contact angle, we observed liquid penetration also above the geometrically expected critical angle. This was not observed for large spheres, as the energy barrier is prohibitively high. Protrusions on the nanoscale slow down imbibition but do not prevent it at long time scales.

We thank Sanghyuk Wooh for SEM measurements. Financial support from an ERC Grant No. 340391 SuPro (H.-J. B.) is acknowledged.

* papadopo@uoi.gr; University of Ioannina, Department of Physics, P.O. Box 1186, GR-45110 Ioannina, Greece

[1] B. Bhushan, Y. C. Jung, and K. Koch, Langmuir 25, 3240 (2009).

[2] M. J. Kreder, J. Alvarenga, P. Kim, and J. Aizenberg, Nat. Rev. Mater. 1, 15003 (2016).

[3] T. Darmanin and F. Guittard, J. Mater. Chem. A 2, 16319 (2014).

[4] N. Shirtcliffe and P. Roach, "Superhydrophobicity for Antifouling Microfluidic Surfaces," in Microfluidic Diagnostics, Methods in Molecular Biology, edited by G. Jenkins and C. D. Mansfield (Humana Press, Totowa NJ, 2013) pp. 269-281.

[5] C.-H. Xue, X.-J. Guo, J.-Z. Ma, and S.-T. Jia, ACS Appl. Mater. Interf. 7, 8251 (2015).

[6] R. Hensel, C. Neinhuis, and C. Werner, Chem. Soc. Rev. 45, 323 (2016).

[7] T. L. Liu and C.-J. C. Kim, Science 346, 1096 (2014).

[8] A. Tuteja, W. Choi, M. Ma, J. M. Mabry, S. A. Mazzella, G. C. Rutledge, G. H. McKinley, and R. E. Cohen, Science 318, 1618 (2007).

[9] These surfaces are also termed superhydrophobic, superamphiphobic, superomniphobic or superhygrophobic, depending on the type of liquids that do not wet the surface. A. Marmur, Soft Matter 8, 6867 (2012).

[10] C. a. E. Hamlett, S. Atherton, N. J. Shirtcliffe, G. McHale, S. Ahn, S. H. Doerr, R. Bryant, and M. I. Newton, Eur. J. Soil Sci. 64, 324 (2013).

[11] N. J. Shirtcliffe, G. McHale, M. I. Newton, F. B. Pyatt, and S. H. Doerr, Appl. Phys. Lett. 89, 094101 (2006).

[12] A. B. D. Cassie and S. Baxter, Trans. Faraday Soc. 40, 546 (1944).

[13] R. N. Wenzel, Ind. Eng. Chem. 28, 988 (1936).

[14] P. Lv, Y. Xue, Y. Shi, H. Lin, and H. Duan, Phys. Rev. Lett. 112, 196101 (2014).

[15] P. Papadopoulos, L. Mammen, X. Deng, D. Vollmer, and H.-J. Butt, Proc. Natl. Acad. Sci. U.S.A. 110, 3254 (2013).

[16] X. Deng, F. Schellenberger, P. Papadopoulos, D. Vollmer, and H.-J. Butt, Langmuir 29, 7847 (2013).
[17] Y. C. Jung and B. Bhushan, Langmuir 24, 6262 (2008).

[18] J. de Ruiter, R. Lagraauw, D. van den Ende, and F. Mugele, Nat. Phys. 11, 48 (2014).

[19] J. V. I. Timonen, M. Latikka, O. Ikkala, and R. H. A. Ras, Nat. Commun. 4, 2398 (2013).

[20] P. S. Brown and B. Bhushan, J. Colloid Interf. Sci. 456, 210 (2015).

[21] M. Paven, R. Fuchs, T. Yakabe, D. Vollmer, M. Kappl, A. N. Itakura, and H.-J. Butt, Adv. Funct. Mater. 26, 4914-4922 (2016).

[22] X. Tian, T. Verho, and R. H. A. Ras, Science 352, 142 (2016).

[23] X. Deng, L. Mammen, H.-J. Butt, and D. Vollmer, Science 335, 67 (2012).

[24] M. Paven, P. Papadopoulos, L. Mammen, X. Deng, H. Sachdev, D. Vollmer, and H.-J. Butt, Pure Appl. Chem. 86, 87 (2014).

[25] S. Ban, E. Wolfram, and S. Rohrsetzer, Colloids Surf. 22, 291 (1987).

[26] J. Bachmann and G. McHale, Eur. J. Soil Sci. 60, 420 (2009).

[27] See Supplemental Material at [url] for confocal microscopy and fluorescent labeling, which includes Ref. [28].

[28] T. Weil, T. Vosch, J. Hofkens, K. Peneva, and K. Mullen, Angew. Chem. Int. Ed. Eng 49, 9068 (2010).

[29] F. Schellenberger, N. Encinas, D. Vollmer, and H.-J. Butt, Phys. Rev. Lett. 116, 096101 (2016).

[30] H.-J. Butt, C. Semprebon, P. Papadopoulos, D. Vollmer, M. Brinkmann, and M. Ciccotti, Soft Matter 9, 418 (2013).

[31] J. T. Korhonen, T. Huhtamaki, O. Ikkala, and R. H. A. Ras, Langmuir 29, 3858 (2013).

[32] A. F. Stalder, T. Melchior, M. Muller, D. Sage, T. Blu, and M. Unser, Colloid Surf. A 364, 72 (2010).

[33] N. Martys, M. Cieplak, and M. O. Robbins, Phys. Rev. Lett. 66, 1058 (1991).

[34] R. Lucas, Kolloid-Zeitschrift 23, 15 (1918).

[35] E. W. Washburn, Phys. Rev. 17, 273 (1921).

[36] M. Alava, M. Dube, and M. Rost, Adv. Phys. 53, 83 (2004).

[37] L. A. Nunes Amaral, A. L. Barabasi, H. A. Makse, and H. E. Stanley, Phys. Rev. E 52, 4087 (1995).

[38] A.-L. Barabasi, G. Grinstein, and M. A. Munoz, Phys. Rev. Lett. 76, 1481 (1996).

[39] Y.-J. Chen, S. Watanabe, and K. Yoshikawa, J. Phys. Chem. C 119, 12508 (2015).

[40] D. Geromichalos, F. Mugele, and S. Herminghaus, Phys. Rev. Lett. 89, 104503 (2002).

[41] D.-S. Lee, Z. Sadjadi, and H. Rieger, Phys. Rev. E 90, 013016 (2014).

[42] E. W. Lemmon, M. O. McLinden, and D. G. Friend, "Thermophysical properties of fluid systems," in NIST Chemistry WebBook, NIST Standard Reference Database Number 69, Eds. P.J. Linstrom and W.G. Mallard, National Institute of Standards and Technology, Gaithersburg MD, 20899, http://webbook.nist.gov, (retrieved August 12, 2015).

[43] R. Schmidt, K. Griesbaum, A. Behr, D. Biedenkapp, H.W. Voges, D. Garbe, C. Paetz, G. Collin, D. Mayer, and H. Hoke, "Hydrocarbons," in Hydrocarbons. Ullmann's Encyclopedia of Industrial Chemistry (Wiley-VCH Verlag GmbH and Co. KGaA, Weinheim, 2014) pp. 1-74.

[44] T. D. Blake and J. M. Haynes, J. Colloid Interface Sci. 
30, 421 (1969).

[45] T. D. Blake, J. Colloid Interface Sci. 299, 1 (2006).

[46] D. Seveno, N. Dinter, and J. De Coninck, Langmuir 26, $14642(2010)$.
[47] D. Seveno, A. Vaillant, R. Rioboo, H. Adao, J. Conti, and J. De Coninck, Langmuir 25, 13034 (2009).

[48] T. Young, Philos. Trans. R. Soc. London 95, 65 (1805).

[49] C. Della Volpe, D. Maniglio, S. Siboni, and M. Morra, Oil and Gas Science and Technology 56, 9 (2001). 